Bio - grafía. Escritos sobre la Biología y su Enseñanza. ISSN 2027-1034

Edición Extraordinaria. p.p. 1378 - 1385

Memorias del IX Encuentro Nacional de Experiencias en Enseñanza de la Biología y la

Educación Ambiental. IV Congreso Nacional de Investigación en Enseñanza de la Biología.

\title{
UN POLO A TIERRA EN LA FORMACIÓN INICIAL DE MAESTROS DE CIENCIAS
} A GROUNDING LINE IN THE INITIAL TRAINING OF TEACHERS OF SCIENCES

\section{Adriana Marcela Torres Durán ${ }^{1}$}

Esta biocrónica da a conocer una experiencia sobre el uso de las microclases como estrategia metodológica de acercamiento a la realidad escolar, como un "polo a tierra", con un grupo de maestros en formación de ciencias naturales, desde el curso de Práctica Pedagógica de la Facultad de Educación de la Universidad de Antioquia. La microclase conocida como microenseñanza, es una herramienta que permite adquirir habilidades docentes con ambientes de aula simulados y planeados de manera progresiva. Se programaron seis sesiones que incluían habilidades para la docencia, siendo acumulativas en cada encuentro. La habilidad sobre el manejo de grupo generó mayores dificultades entre los participantes, posiblemente por considerarlo un aparente factor sencillo de abordar y que con la experiencia que algunos tenían, podrían resolverlo. Con esta estrategia metodológica se logró la observación y autoevaluación del desempeño de los maestros en formación, en ambientes controlados y de tipo laboratorio de enseñanza, lo cual facilitó el aprendizaje de habilidades para la docencia y el reconocimiento de la importancia de la reflexión pedagógica como parte de la formación inicial y continua.

\section{A MODO DE INTRODUCCIÓN}

La innegable importancia de las prácticas en la formación inicial docente y los resultados de la investigación de Correa (2011), señalan que "las prácticas no satisfacen las expectativas de desarrollo de competencias" (p.78). Es así como se evidencia la necesidad junto a mi interés personal por revisar estrategias de apoyo y acompañamiento en el curso de Práctica Pedagógica, que oriento en la Licenciatura en Educación Básica con énfasis en Ciencias Naturales y Educación Ambiental en Puerto Berrío, Antioquia. Una de las estrategias que implementé con un grupo fue el diseño, ejecución y análisis de microclases, desde la base teórica de la microenseñanza según Allen y Ryan (1968).

En mi formación inicial como docente tuve la oportunidad de vivenciar las microclases, como parte del plan del curso de práctica pedagógica en la Universidad del Tolima, estrategia que considero valiosa en el proceso de adquisición de habilidades docentes y que aporta a la reflexión pedagógica generada por la autoevaluación y autorregulación del maestro en formación. Sanmartí (2001), resalta la importancia de formar a los futuros profesionales en la reflexión, que ha de proporcionar criterios para la toma de decisiones y

\footnotetext{
${ }^{1}$ Catedrática de Práctica Pedagógica de la Licenciatura en Educación Básica con énfasis en Ciencias Naturales - Seccional Magdalena Medio, Puerto Berrío - Universidad de Antioquia. Docente de la Secretaría de Educación de Medellín. Estudiante del Doctorado en Educación e integrante del Grupo PiEnCias, UdeA. Correo: amarcela.torres@udea.edu.co
} 


\title{
Bio - grafía. Escritos sobre la Biología y su Enseñanza. ISSN 2027-1034
}

\section{Edición Extraordinaria. p.p. 1378 - 1385}

\author{
Memorias del IX Encuentro Nacional de Experiencias en Enseñanza de la Biología y la \\ Educación Ambiental. IV Congreso Nacional de Investigación en Enseñanza de la \\ Biología.
}

en la autonomía del profesor. Al mismo tiempo, evita que los profesores en formación se acostumbren a generalizar a partir de la propia experiencia, sin tener en cuenta los conocimientos del campo profesional. Así, la reflexión in situ es base fundamental en la formación docente.

Un modelo de formación de profesores necesita que los estudiantes construyan un sistema efectivo de autorregulación (cognitiva, ética y emocional) y que adquieran la mejor autonomía posible para orientar no sólo su proceso de formación inicial, sino también el de formación permanente (Sanmartí, 2001). Se trata de conseguir que el futuro enseñante sea gestor de su propia práctica y que aprenda a aprender a lo largo de toda su vida profesional (Sacristán, 1983, citado por Sanmartí, 2001). Teniendo en cuenta lo anterior y aprobada la propuesta ante el comité de prácticas, la microenseñanza fue una de las estrategias metodológicas usadas en el curso de Práctica Pedagógica I.

Mis estudiantes, que serán llamados de aquí en adelante como maestros en formación, se mostraron receptivos, un poco ansiosos y luego de una presentación que incluía la mención de expectativas de la práctica, empezaron las preguntas:
"¿Profesora, es que yo nunca he ido a un colegio a dar clases y sinceramente me da mucho miedo, es posible que me deje en primaria?"; “¿Profe, yo soy normalista y estoy trabajando en provisionalidad, será posible que mi trabajo me sirva de práctica, es que casi no tengo tiempo para hacer práctica en otro lugar?"; "¿La verdad, yo no entiendo muy bien eso de las microclases, van a venir niños a los seminarios?", "¿A mí me inquieta ir a dar clases porque siento que aún soy muy tímida, en este semestre puedo hacer sólo observación de clase?”; “¿Profe, yo puedo escoger mi centro de práctica?".

Estas preguntas hacen evidente un panorama que esperaba. Es conocido entre maestros que la práctica constituye una de las experiencias de formación más importantes en nuestra profesión, que puede tener diferentes matices; por esta razón, comprendo que la práctica pedagógica es una asignatura diferente, que genera inquietudes, miedos, ansiedad, alegrías y por qué no decirlo, hasta dudas con la escogencia de profesión. Cuando presenté mi propuesta, hice énfasis en la importancia de las habilidades para la docencia, que a pesar de ser contempladas desde una mirada artesanal del profesor, las considero vitales en la formación de maestros.

Desde mi experiencia durante doce años como profesora de Ciencias Naturales en Básica Secundaria, en colegios privados y oficiales, he visto la necesidad de incluir en la formación de profesores, las habilidades docentes que le permitan al maestro en formación lograr un mejor desempeño en el aula y una adecuada preparación emocional. Además, como maestra cooperadora, he encontrado que las recientes generaciones de maestros, presentan dificultades en asuntos de gestión del aula y actitudes para la resolución de conflictos. Por tanto, considero que la microenseñanza puede aportar al fortalecimiento de una estrategia de formación integral de los maestros, considerándola una herramienta pedagógica que va más allá de reforzar métodos o hacer evaluación de control. Las microclases sirven para que los profesores en formación se encuentren a ellos mismos en relación con sus educandos. 


\title{
Bio - grafía. Escritos sobre la Biología y su Enseñanza. ISSN 2027-1034
}

\section{Edición Extraordinaria. p.p. 1378 - 1385}

\author{
Memorias del IX Encuentro Nacional de Experiencias en Enseñanza de la Biología y la \\ Educación Ambiental. IV Congreso Nacional de Investigación en Enseñanza de la \\ Biología.
}

La Práctica Pedagógica en cada Universidad tiene sus particularidades, pero en general, en todas se busca que los maestros en formación tengan un acercamiento a las dinámicas de la escuela, a las relaciones y conexiones que se establecen entre los actores, así como al trabajo de aula cuando el maestro en formación pasa de ser estudiante a profesor. Este documento presenta la experiencia con un grupo de siete maestros en formación, de los cuales tres son profesores en ejercicio, que participaron en el curso de práctica pedagógica I, que tiene dos componentes: docencia e investigación. En el componente docencia, la microenseñanza fue una de las estrategias usadas en clase.

Pero... ¿Para qué sirve la microenseñanza? ¿No están pasadas de moda? La microenseñanza fue una estrategia usada en muchas instituciones de formación de maestros, incluyendo algunas Escuelas Normales; sin embargo, se ha dejado de considerar en los seminarios de formación de maestros por considerarse un "entrenamiento" que beneficia poco a los docentes y le da una concepción instrumentalista a la educación. También se considera que este ejercicio simplifica la complejidad del proceso de enseñanza y se teme que el seminario de práctica quede reducido a esta actividad como única, dejando de lado la revisión teórica, la reflexión y especialmente la investigación. En revisión de literatura, se encuentra sobre la microenseñanza que por primera vez en 1963, un grupo de investigadores y de maestros de la Universidad de Stanford (Allen y Ryan, 1968) la diseñaron para abatir muchas de las deficiencias de los programas tradicionales de la educación de maestros, y para acrecentar el entendimiento del proceso que se establece entre el profesor y la enseñanza (Pelberg, 1972).

En los años setenta recibe los aportes de Piaget, Vigotsky, entre otros, teniendo en cuenta que cada estudiante estructura su conocimiento estableciendo relaciones racionales y significativas con el mundo. En la década del 90 ya es considerada como una práctica reflexiva y de instrospección acerca del propio desempeño. Se brinda al docente la oportunidad de desarrollar su actividad frente a otros compañeros, quienes actúan de observadores capaces de proporcionarles retroinfomación valiosa para mejorar (Hernández, 2011).

La microenseñanza implica la simulación de experiencias didácticas de los estudiantes que se forman como profesores. Esto requiere una simplificación del tiempo, del número de alumnos y de la selección de contenidos curriculares con el fin de lograr competencias pedagógicas en los aprendices o docentes en servicio a través de instrumentos de evaluación en condiciones controladas. Según Sacristán (2008), su finalidad es "contribuir al proceso de formación del profesorado haciendo énfasis en conductas precisas y observables que se espera, muestre el profesor que se está formando" (p.367). Pretende dos objetivos complementarios: la formación de la persona y la del grupo (Postic y De Ketele, 1992, citado por Bernal y Cordero, 2013).

De la misma manera, Guillermo (1997), considera conveniente e importante utilizar la microenseñanza para probar estrategias diferentes de enseñanza, la supervisión del profesor en el aula y la autoevaluación como el principal elemento para el cambio. Además, es una técnica que permite a los profesores practicar las habilidades docentes 


\title{
Bio - grafía. Escritos sobre la Biología y su Enseñanza. ISSN 2027-1034
}

\section{Edición Extraordinaria. p.p. 1378 - 1385}

\author{
Memorias del IX Encuentro Nacional de Experiencias en Enseñanza de la Biología y la \\ Educación Ambiental. IV Congreso Nacional de Investigación en Enseñanza de la \\ Biología.
}

en un escenario seguro, con las complejidades normales del aula pero minimizadas, y en el que se le proporciona retroalimentación inmediata. En la década de los 80s, la formación inicial adquiere rango universitario en una reforma neocapitalista y se crean programas de formación permanente del profesorado, enmarcados predominantemente en modalidades propias del modelo de entrenamiento y de observación/evaluación (como la microenseñanza).También se busca una auto-observación a voluntad para que, con la debida orientación, el maestro en formación pueda autocorregirse y mejorar profesionalmente en unas condiciones óptimas y sin perjuicio en el aprendizaje de los estudiantes (Ortuño, 1973).

Para algunos profesores universitarios, la microenseñanza es una estrategia que se usó y funcionó en su época, pero que en pleno siglo XXI está pasada de moda. Y cuando se habla de habilidades o competencias docentes esto lleva a pensar que, "si se les presenta planes con base a modelos de conducta reducido, así responderán y lo mismo si se aplican competencias operativas o académicas" (Barnett, 2001 p. 2). Pero, cuando las habilidades profesionales incluyen la planificación, la identificación y el uso de diferentes métodos y técnicas, la comunicación efectiva, la atención de los estudiantes y el conocimiento de sus necesidades, la gestión de clase, la gestión del tiempo, la clasificación y la evaluación, las microclases aportan al saber práctico del docente de aula; este saber aportará en situaciones difíciles de la clase, que no sólo deben abordarse desde la teoría, porque corresponden a la compleja sociedad que tenemos representada día a día en nuestros estudiantes.

Además, algunas investigaciones nacionales e internacionales indican que la microenseñanza es eficaz para desarrollar actitudes positivas hacia la profesión de enseñar y para ayudar a los estudiantes a adquirir ciertas habilidades fundamentales (Uşun y Zorlubaş, 2007, citado por Barnett, 2001). También, aumenta su confianza en sí mismos al darles conciencia de los valores, actitudes y suposiciones del aprendizaje y de sus identidades académicas.

Con esta experiencia se buscó rescatar las bondades de la microenseñanza y fortalecer las habilidades docentes. Además de acompañar en el proceso de autoevaluación y autorregulación de cada maestro en formación, a partir de la retroalimentación (Feedback de la asesora y de los compañeros docentes) de tal manera que se lograra observar aquellos aspectos positivos en su desempeño en clase, y al mismo tiempo identificar aquello que requiere mejorar y que luego sería consignado en sus diarios pedagógicos.

Microclases en Práctica. Las clases de práctica pedagógica I están ubicadas en el plan de estudios en el nivel $8^{\circ}$. En las regiones de la Universidad, están planeadas para dos seminarios presenciales cada quince días, por organización distribuí en cada encuentro un seminario para docencia y el otro para investigación. Las sesiones para trabajar las microclases se planearon con base en el libro Habilidades para la docencia de Zarzar (2006); así como de los textos de Vaello Resolución de conflictos en el aula (2002), Habilidades sociales en el aula (2005) Estrategias para la mejora de la gestión de aula (2007) y Cómo dar clase a los que no quieren (2007), Videos de apoyo a la enseñanza, entre otros. Cada sesión contaba con un tema pedagógico o didáctico y una habilidad docente (Figura 1). 
Bio - grafía. Escritos sobre la Biología y su Enseñanza. ISSN 2027-1034

Edición Extraordinaria. p.p. 1378 - 1385

Memorias del IX Encuentro Nacional de Experiencias en Enseñanza de la Biología y la

Educación Ambiental. IV Congreso Nacional de Investigación en Enseñanza de la Biología.

En la primera sesión se dieron a conocer las características que debía cumplir una microclase como simulación de una clase que se daría en el Centro de Práctica, además explicitar lo trabajado en el seminario según tema y habilidad. Por ejemplo, en la primera sesión se dieron pautas respecto a la estructura de una clase (inicio, desarrollo y cierre) y sobre el manejo de la voz y la postura corporal, se entregaron documentos de apoyo, que consistían en guías de trabajo y videos para que los maestros en formación planearan su primera microclase para el siguiente seminario de docencia.

En el segundo seminario de docencia se iniciaron las microclases por cada maestro en formación. Cada uno contaba con 2 minutos para presentar su microclase, 12 minutos para realizarla y hasta 10 minutos para hacer su autoevaluación y recibir retroalimentación por parte de sus compañeros y la asesora. La planeación previamente elaborada, correspondía a la estructura de los Estándares Básicos de Competencias en Ciencias Naturales (Guía Formar en Ciencias del Ministerio de Educación Nacional, 2004). Además, de tener en cuenta características contextuales de sus centros de práctica.

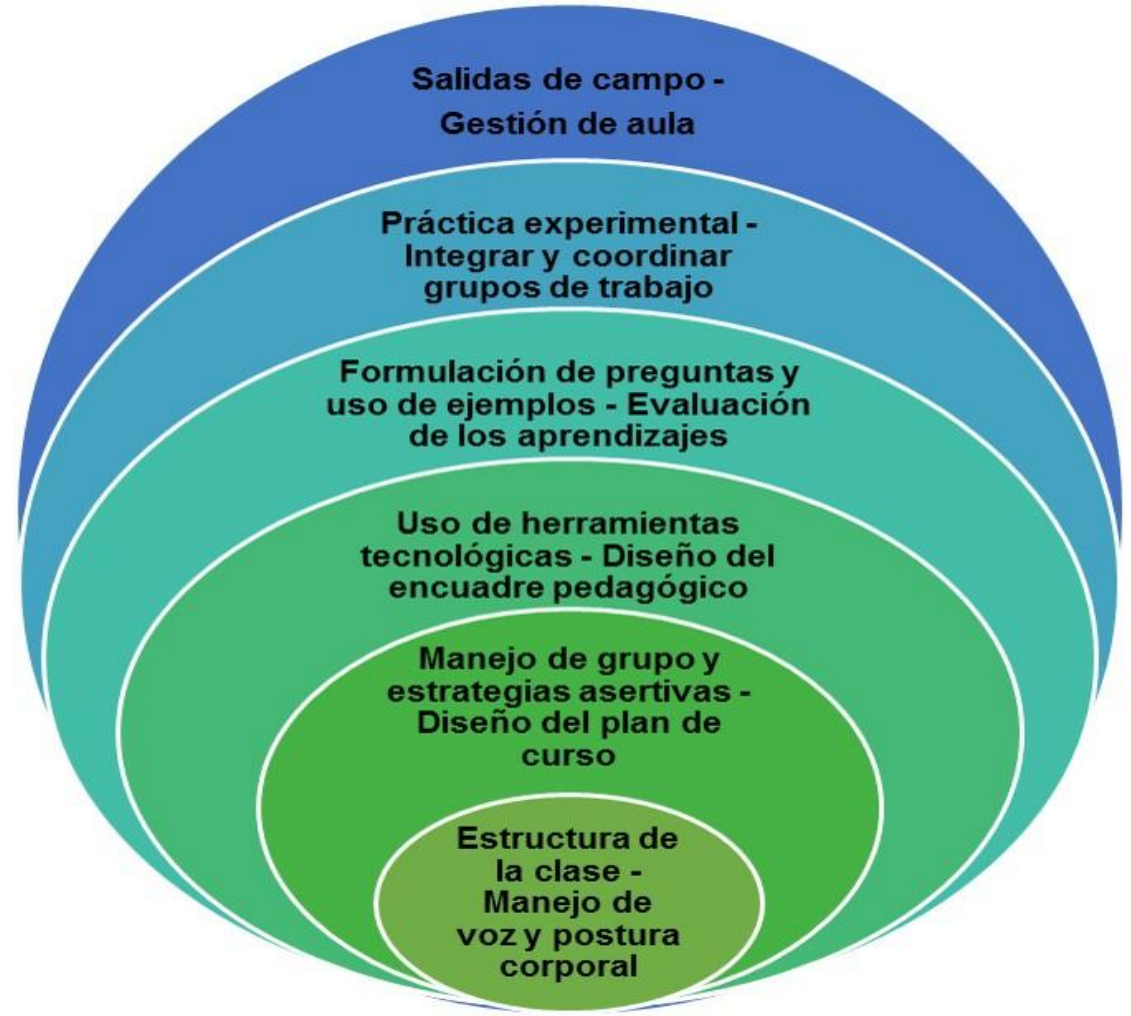

Figura 1. Temas y habilidades trabajados en seis sesiones de la práctica pedagógica con la metodología de Microclases. Elaboración propia.

Los temas y habilidades fueron acumulativos y graduales, de esta manera en cada microclase se tenían en cuenta todas las habilidades trabajadas en el seminario anterior. Mediante acuerdo con los maestros en formación, se estableció que dos de las microclases serían grabadas con la intención de convertirse en recurso útil para la 
Bio - grafía. Escritos sobre la Biología y su Enseñanza. ISSN 2027-1034

Edición Extraordinaria. p.p. 1378 - 1385

Memorias del IX Encuentro Nacional de Experiencias en Enseñanza de la Biología y la

Educación Ambiental. IV Congreso Nacional de Investigación en Enseñanza de la Biología.

autoevaluación y la búsqueda de estrategias para el mejoramiento. Estas grabaciones no fueron publicadas en ningún sitio web ni red social.

La evaluación fue de tipo formativa, incluyendo la autoevaluación, la coevaluación y la heteroevaluación en cada sesión, teniendo en cuenta los avances y mejoras. En el grupo se establecieron acuerdos para asumir una actitud de respeto hacia el maestro que desarrollaba la microclase, en ocasiones asumieron roles específicos como el hiperactivo, el preguntón, el charlatán, entre otros, buscando hacer un poco más real la simulación.

Aprendizajes y relaciones. Las microclases fueron consideradas por los maestros en formación como una estrategia de aula para fortalecer habilidades básicas y especialmente para adquirir mayor confianza en su quehacer docente. En cada sesión los participantes mostraban más seguridad pero persistían en la dificultad del manejo del tiempo.

Con el uso de esta estrategia se lograron relaciones entre los documentos trabajados en la relación entre teoría y práctica, formación docente y sus experiencias como maestros en formación en los Centros de Práctica. Reconocieron la oportunidad de hacer reflexión pedagógica en cada sesión a partir del ejercicio de evaluación formativa y de sus escritos en el diario pedagógico. En la figura 2, se muestra también que al realizar de manera paralela las microclases con su práctica de inmersión genera un acompañamiento necesario para la autorregulación, la reflexión pedagógica que redunda en el fortalecimiento en la formación docente.

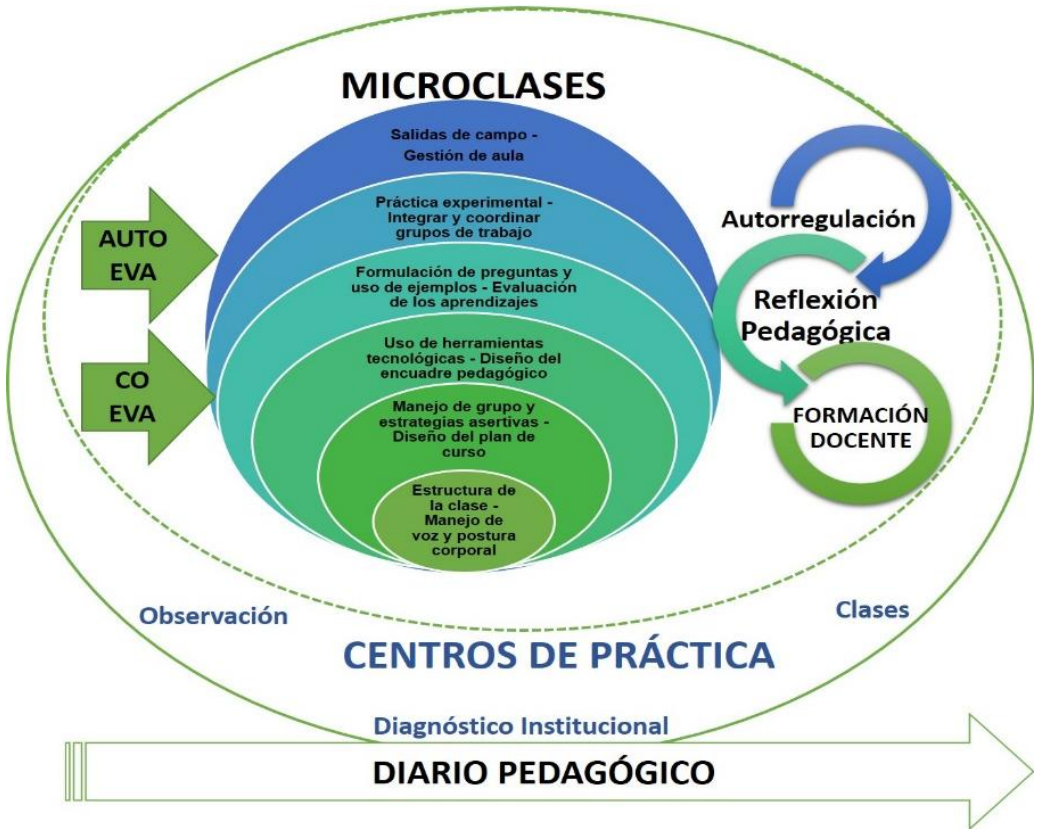

Figura 2. Relaciones con el uso de microclases en la Práctica Pedagógica. Elaboración propia. 
Bio - grafía. Escritos sobre la Biología y su Enseñanza. ISSN 2027-1034

Edición Extraordinaria. p.p. 1378 - 1385

Memorias del IX Encuentro Nacional de Experiencias en Enseñanza de la Biología y la Educación Ambiental. IV Congreso Nacional de Investigación en Enseñanza de la Biología.

Por otro lado, en el uso de recursos tecnológicos, diseño de la práctica experimental y de la salida pedagógica los maestros en formación mostraron un muy buen desempeño. Lograron planear buenas clases y en la ejecución mostraron apropiación de los conocimientos científicos. Por el contrario, la microclase sobre manejo de grupo, generó dificultades entre los maestros, no sólo por falta de estrategias asertivas para las diferentes situaciones simuladas como agresión entre estudiantes y roles de estudiantes hiperactivos y retadores, sino también porque sintieron desvalorada su clase al ver frustrada su planeación por tener que resolver conflictos, encontrándose a su vez como una fortaleza, porque a veces la realidad educativa es más compleja de lo que en teoría se menciona.

En palabras de los maestros en formación, podríamos concluir que "las microclases fortalecen la planeación de las clases de ciencias naturales y el manejo efectivo del tiempo"; además "se logran identificar estrategias para el manejo de grupos y casos o situaciones de clase asociadas con indisciplina, y en general pensar la clase como profesor y repensar la manera como comprendo la enseñanza de las ciencias y su aprendizaje desde diferentes enfoques". "Lo que más me gustó fue sentir que aunque todos teníamos diferentes dificultades, entre todos aportábamos a nuestro crecimiento personal y profesional como maestros, esto fue como nuestro Polo a Tierra".

\section{REFERENCIAS BIBLIOGRÁFICAS}

Allen, D. y Ryan, K. (1969). Microteaching [Microenseñanza]. Reading, Massachusetts: Addison-Wesley Publishing Company.

Barnett, R. (2001). Los límites de la competencia. El conocimiento, la educación superior y la sociedad, Barcelona: Gedisa.

Bernal, J. y Cordero, M. (2013). Aplicando la técnica de microenseñanza en el Modelo de Aprendizaje Basado en Competencias (MABC). Eleventh LACCEI Latin American and Caribbean Conference for Engineering and Technology. Cancún, México. Extraído el 15 de mayo de 2016, de http://www.laccei.org/LACCEI2013Cancun/RefereedPapers/RP257.pdf

Correa, E. (2011). La práctica docente: una oportunidad de desarrollo profesional. Revista Perspectiva Educacional, Vol 50, № 2.

Guillermo, M.C. (1997). Microenseñanza: ¿Una técnica vigente para el desarrollo de habilidades docentes? Educación y Ciencia. 1 (2). 59 - 68. Extraído el 6 de junio de 2016, www.educacionyciencia.org/index.php/educacionyciencia/article/download/120/pdf

Hernández, J.P. (2011). Formación docente, continua y Microenseñanza. Extraído el 21 de mayo de 2017, de http://educacinenlasociedaddelconocimiento.blogspot.com.co/

Ortuño, v. (1973). Microenseñanza: qué es y qué significa la microenseñanza dentro de las innovaciones educativas. Revista de Educación, Extraído el 25 de marzo de 
Bio - grafía. Escritos sobre la Biología y su Enseñanza. ISSN 2027-1034

Edición Extraordinaria. p.p. 1378 - 1385

Memorias del IX Encuentro Nacional de Experiencias en Enseñanza de la Biología y la

Educación Ambiental. IV Congreso Nacional de Investigación en Enseñanza de la Biología.

2016, de https://www.mecd.gob.es/dctm/revista-de-educacion/1973219/1972re219microensenanza.pdf?documentld=0901e72b818218b8

Sanmartí, N. (2001). Enseñar a enseñar ciencias en secundaria: un reto muy completo. Revista Interuniversitaria de Formación del Profesorado. 40, p. 31-48.

Pelberg, A. (1972). Microenseñanza: Un Innovador Procedimiento de Laboratorio para Mejorar la Enseñanza y el Entrenamiento de Profesores. Higher Education Journal, 1(2), 1-15.

Sacristán, G.J. (comp.) (2008). Educar por competencias, ¿qué hay de nuevo?, Madrid: Morata.

Vaello, J. (2003): Resolución de conflictos en el aula. Madrid: Santillana.

(2005): Habilidades sociales en el aula. Madrid: Santillana.

(2007): Cómo dar clase a los que no quieren. Madrid: Santillana.

Zarzar, C. (2006). Habilidades básicas para la docencia. México: La Patria. 152 p. 